\title{
Energy-Efficient Process Allocation Algorithms in Peer-to-Peer Systems
}

\author{
Ailixier Aikebaier ${ }^{2}$, Tomoya Enokido ${ }^{1}$, and Makoto Takizawa ${ }^{2}$ \\ 1 Rissho University, Japan \\ eno@ris.ac.jp \\ 2 Seikei University, Japan \\ alisher.akber@computer.org, \\ makoto.takizawa@st.seikei.ac.jp
}

\begin{abstract}
Information systems are composed of various types of computers interconnected in networks. In addition, information systems are being shifted from the traditional client-server model to the peer-to-peer (P2P) model. The P2P systems are scalable and fully distributed without any centralized coordinator. It is getting more significant to discuss how to reduce the total electric power consumption of computers in information systems in addition to developing distributed algorithms to minimize the computation time. In this paper, we do not discuss the micro level like the hardware specification of each computer. We discuss a simple model to show the relation of the computation and the total power consumption of multiple peer computers to perform types of processes at macro level. We also discuss algorithms for allocating a process to a computer so that the deadline constraint is satisfied and the total power consumption is reduced.
\end{abstract}

\section{Introduction}

Information systems are getting scalable so that various types of computational devices like server computers and sensor nodes [1] are interconnected in types of networks like wireless and wired networks. Various types of distributed algorithms [6] are so far developed, e.g. for allocating computation resources to processes and synchronizing multiple conflicting processes are discussed to minimize the computation time and response time, maximize the throughput, and minimize the memory space. On the other hand, the green IT technologies [4] have to be realized in order to reduce the consumptions of natural resources like oil and resolve air pollution on the Earth. In information systems, total electric power consumption has to be reduced. Various hardware technologies like low-power consumption CPUs [2]3] are now being developed. Biancini et al. [8] discuss how to reduce the power consumption of a data center with a cluster of homogeneous server computers by turning off servers which are not required for executing a collection of web requests. Various types of algorithms to find required number of servers in homogeneous and heterogeneous servers are discussed [59]. In wireless sensor networks [1], routing algorithms to reduce the power consumption of the battery in a sensor node are discussed.

In this paper, we consider peer-to-peer (P2P) overlay networks [7] where computers are in nature heterogeneous and cannot be turned off by other persons different from 
the owners. In addition, the P2P overlay networks are scalable and fully distributed with no centralized coordination. Each peer has to find peers which not only satisfy QoS requirement but also spend less electric power. First, we discuss a model for performing processes on a computer. Then, we measure how much electric power a type of computers spends to perform a Web application process. Next, we discuss a simple power consumption model for performing a process in a computer based on the experiments with servers and personal computers. In the simple model, each computer consumes maximally the electric power if at least one process is performed. Otherwise, the computer consumes minimum electric power, i.e. in idle state. The simple model shows personal computers with one CPU independently of the number of cores, according to our experiment.

A request to perform a process like a Web page request is allocated to one of the computers in the network. We discuss a laxity-based allocation algorithm to reduce not only execution time but also power consumption in a collection of computers. In the laxity-based algorithm, processes are allocated to computers so that the deadline constraints are satisfied based on the laxity concept.

In section 2, we present a systems model for performing a process on a computer. In section 3, we discuss a simple power consumption model obtained from the experiment. In section 4, we discuss how to allocate each process with a computer to reduce the power consumption. In section 5, we evaluate the process allocation algorithms.

\section{Computation Model}

\subsection{Normalized Computation Rate}

A system $S$ is includes a set $C$ of computers $c_{1}, \ldots, c_{n}(n \geq 1)$ interconnected in reliable networks. A user issues a request to perform a process like a Web page request. The process is performed on one computer. There are a set $P$ of application processes $p_{1}$, $\ldots, p_{m}(m \geq 1)$. A term process means an application process in this paper. We assume each process $p_{s}$ can be performed on any computer in the computer set $C$. A user issues a request to perform a process $p_{s}$ to a load balancer $K$. For example, a user issues a request to read a web page on a remote computer. The load balancer $K$ selects one computer $c_{i}$ in the set $C$ for a process $p_{s}$ and sends a request to the computer $c_{i}$. On receipt of the request, the process $p_{s}$ is performed on the computer $c_{i}$ and a reply, e.g. Web page is sent back to the requesting user. Requests from multiple users are performed on a computer $c_{i}$. A process being performed at time $t$ is referred to as current. A process which already terminates before time $t$ is referred to as previous. Let $P_{i}(t)(\subseteq P)$ be a set of current processes on a computer $c_{i}$ at time $t . N_{i}(t)$ shows the number of the current processes in the set $P_{i}(t), N_{i}(t)=\left|P_{i}(t)\right|$. Let $P(t)$ show a set of all current processes on computers in the system $S$ at time $t, \cup_{i=1, \ldots, n} P_{i}(t)$.

Suppose a process $p_{s}$ is performed on a computer $c_{i}$. Here, $T_{i s}$ is the total computation time of $p_{s}$ on $c_{i}$ and $\min T_{i s}$ shows the computation time $T_{i s}$ where a process $p_{s}$ is exclusively performed on $c_{i}$, i.e. without any other process. Hence, $\min T_{i s} \leq$ $T_{i s}$ for every process $p_{i}$. Let $\max T_{s}$ and $\min T_{s}$ be $\max \left(\min T_{1 s}, \ldots, \min T_{n s}\right)$ and $\min \left(\min T_{1 s}, \ldots, \min T_{n s}\right)$, respectively. If a process $p_{s}$ is exclusively performed on the fastest computer $c_{i}$ and the slowest computer $c_{j}, \min T_{s}=\min T_{i s}$ and $\max T_{s}=$ 
$\min T_{j s}$, respectively. A time unit $(t u)$ shows the minimum time to perform a smallest process. We assume it takes at least one time unit [tunit] to perform a process on any computer, i.e. $1 \leq \operatorname{minT}_{s} \leq \max T_{s}$. The average computation rate $(A C R) F_{i s}$ of a process $p_{s}$ on a computer $c_{i}$ is defined as follows:

$$
F_{i s}=1 / T_{i s}[1 / \mathrm{tu}] \text {. }
$$

Here, $0<F_{i s} \leq 1 / \operatorname{minT}_{i s} \leq 1$. The maximum $A C R \max F_{i s}$ is $1 / \min _{i s} . F_{i s}$ shows how many percentages of the total amount of computation of a process $p_{s}$ are performed for one time unit. $\max F_{s}=\max \left(\max F_{1 s}, \ldots, \max F_{n s}\right) . \min F_{s}=\min \left(\max F_{1 s}, \ldots\right.$, $\left.\max F_{n s}\right) . \max F_{s}$ and $\min F_{s}$ show the maximum $A C R \mathrm{~s} \max F_{i s}$ and $\max F_{j s}$ for the fastest computer $c_{i}$ and the slowest computer $c_{j}$, respectively.

The more number of processes are performed on a computer $c_{i}$, the longer it takes to perform each of the processes on the computer $c_{i}$. Let $\alpha_{i}(t)$ indicate the degradation rate of a computer $c_{i}$ at time $t\left(0 \leq \alpha_{i}(t) \leq 1\right)[1 / \mathrm{tu}] . \alpha_{i}\left(t_{1}\right) \leq \alpha_{i}\left(t_{2}\right) \leq 1$ if $N_{i}\left(t_{1}\right) \leq$ $N_{i}\left(t_{2}\right)$ for every pair of different times $t_{1}$ and $t_{2}$. We assume $\alpha_{i}(t)=1$ if $N_{i}(t) \leq 1$ and $\alpha_{i}(t)<1$ if $N_{i}(t)>1$. Suppose it takes 50 [msec] to exclusively perform a process $p_{s}$ on a computer $c_{i}$. Here, $\min T_{i s}=50$. Here, $F_{i s}=\max F_{i s}=1 / 50[1 / \mathrm{msec}]$. Suppose it takes $75[\mathrm{msec}]$ to perform the process $p_{s}$ while other processes are performed on the computer $c_{i}$. Here, $F_{i s}=1 / 75[1 / \mathrm{msec}]$. Hence, $\alpha_{i}(t)=50 / 75=0.67[1 / \mathrm{msec}]$. We define the normalized computation rate $(N C R) f_{i s}(t)$ of a process $p_{s}$ on a computer $c_{i}$ at time $t$ as follows:

$$
f_{i s}(t)=\left\{\begin{array}{l}
\alpha_{i}(t) \cdot \max F_{i s} / \max F_{s}[1 / \mathrm{tu}] \\
\alpha_{i}(t) \cdot \operatorname{minT}_{s} / \operatorname{minT}_{i s}[1 / \mathrm{tu}]
\end{array}\right.
$$

For the fastest computer $c_{i}, f_{i s}(t)=1$ if $\alpha_{i}(t)=1$, i.e. $N_{i}(t)=1$. If a computer $c_{i}$ is faster than another computer $c_{j}$ and the process $p_{s}$ is exclusively performed on $c_{i}$ and $c_{j}$ at time $t_{i}$ and $t_{j}$, respectively, $f_{i s}\left(t_{i}\right)>f_{j s}\left(t_{j}\right)$. If a process $p_{s}$ is exclusively performed on a computer $c_{i}, \alpha_{i s}(t)=1$ and $f_{i s}(t)=\max F_{i s} / \max F_{s}$. The maximum NRC $\max f_{i s}$ shows $\max F_{i s} / \max F_{s} .0 \leq f_{i s}(t) \leq \max f_{i s} \leq 1$. The NCR $f_{i s}(t)$ shows how many steps of a process $p_{s}$ are performed on a computer $c_{i}$ at time $t$. The average computation rate $(A C R) F_{i s}$ depends on the size of the process $p_{s}$ while $f_{i s}(t)$ depends on the speed of the computer $c_{i}$.

Next, suppose that a process $p_{s}$ is started and terminated on a computer $c_{i}$ at time $s t_{i s}$ and $e t_{i s}$, respectively. Here, the total computation time $T_{i s}$ is $e t_{i s}-s t_{i s}$. The following formulas hold for the degradation rate $\alpha_{i}(t)$ and $\operatorname{NCR} f_{i s}(t)$ :

$$
\begin{gathered}
\int_{s t_{i s}}^{e t_{i s}} \frac{\alpha_{i}(t)}{\operatorname{minT}_{i s}}=1 \\
\int_{s t_{i s}}^{e t_{i s}}\left(f_{i s}(t)\right) d t=\operatorname{minT}_{s} \cdot \int_{s t_{i s}}^{e t_{i s}} \frac{\alpha_{i}(t)}{\operatorname{minT}_{i s}}=\operatorname{minT}_{s}
\end{gathered}
$$

If there is no other process, i.e. $\alpha_{i}(t)=1$ on the computer $c_{i}, f_{i s}(t)=\max F_{i s} / \max F_{s}=$ $\min T_{s} / \operatorname{minT}_{i s}$. Hence, $T_{i s}=e t_{i s}-s t_{i s}=m i n T_{i s}$. If other processes are performed, $T_{i s}=e t_{i s}-s t_{i s}>\min T_{i s}$. Here, $\min T_{s}$ shows the total amount of computation to be performed by the process $p_{s}$. 
Figure 1 shows the NCRs $f_{i s}(t)$ and $f_{j s}(t)$ of a process $p_{s}$ which are exclusively performed on a pair of computers $c_{i}$ and $c_{j}$, respectively. Here, the computer $c_{i}$ is the fastest in the computer set $C$. The NCR $f_{i s}(t)=\max _{i s}=1$ for $s t_{i s} \leq t \leq e t_{i s}$ and $T_{i s}=e t_{i s}-s t_{i s}=\min T_{s}$. On the slower computer $c_{j}, f_{j s}(t)=\max f_{j s}<1$ and $T_{j s}$ $=e t_{j s}-s t_{j s}>\operatorname{minT} T_{s}$. Here, $\max f_{i s} \cdot \operatorname{minT} T_{i s}=\operatorname{minT} T_{s}=\max _{j s} \cdot \operatorname{minT} T_{j s}$ from the equation (4). The areas shown by $f_{i s}(t)$ and $f_{j s}(t)$ have the same size $\min T_{s}(=$ $\left.T_{i s}\right)$. Figure 2 shows the NCR $f_{i s}(t)$ of a process $p_{s}$ on a computer $c_{i}$ at time $t$, where multiple precesses are performed concurrently with the process $p_{s} . f_{i s}(t)$ is smaller than $\max f_{i s}$ if other processes are concurrently performed on the computer $c_{i}$. Here, $T_{i s}=$ $e t_{i s}-s t_{i s}>\operatorname{minT} T_{s}$ and $\int_{s t_{i s}}^{e t_{i s}} f_{i s}(t) d t=\operatorname{minT} T_{s}$.

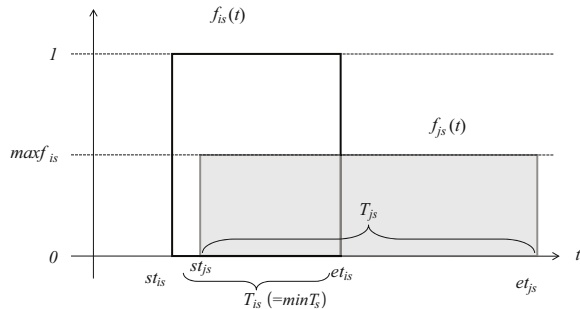

Fig. 1. Normalized computation rates (NCRs)

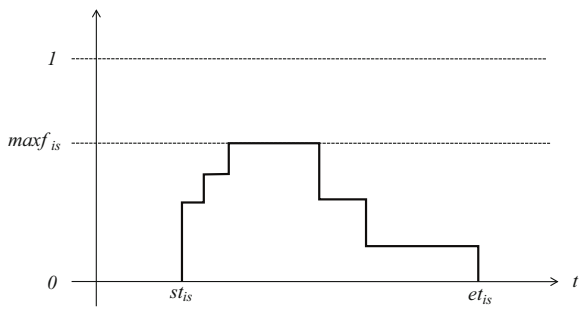

Fig. 2. Normalized computation rate $f_{i s}(t)$

Next, we define the computation laxity $L_{i s}(t)[\mathrm{tu}]$ of a process $p_{s}$ on a computer $c_{i}$ at time $t$ as follows:

$$
L_{i s}(t)=\operatorname{minT}_{s}-\int_{s t_{i s}}^{t}\left(f_{i s}(x)\right) d x .
$$

The laxity $L_{i s}(t)$ shows how much computation the computer $c_{i}$ has to spend to perform up a process $p_{s}$ at time $t . L_{i s}\left(s t_{i s}\right)=\operatorname{minT} T_{s}$ and $L_{i s}\left(e t_{i s}\right)=0$. If the process $p_{s}$ would be exclusively performed on the computer $c_{i}$, the process $p_{s}$ is expected to terminate at time $e t_{i s}=t+L_{i s}(t)$.

\subsection{Simple Computation Model}

There are types of computers with respect to the performance. First, we consider a simple computation model. In the simple computation model, a computer $c_{i}$ satisfies the following properties:

\section{[Simple computation model]}

1. $\max f_{i s}=\max f_{i u}$ for every pair of different processes $p_{s}$ and $p_{u}$ performed on a computer $c_{i}$.

2.

$$
\sum_{p_{s} \in P_{i}(t)} f_{i s}(t)=\max f_{i}
$$


The maximum normalized computation rate (NCR) $\max f_{i}$ of a computer $c_{i}$ is $\max f_{i s}$ for any process $p_{s}$. This means, the computer $c_{i}$ is working to perform any process with the maximum clock frequency. $P_{i}(t)$ shows a set of processes being performed on a computer $c_{i}$ at time $t$. In the simple computation model, we assume the degradation factor $\alpha_{i}(t)=1$.

On a computer $c_{i}$, each process $p_{s}$ starts at time $s t_{i s}$ and terminates at time $e t_{i s}$. We discuss how the NCR $f_{i s}(t)$ of each process $p_{s}$ changes in presence of multiple precesses on a computer $c_{i}$. A process $p_{s}$ is referred to as precedes another process $p_{u}$ on a computer $c_{i}$ if $e t_{i s}<s t_{i u}$. A process $p_{s}$ is interleaved with another process $p_{u}$ on a computer $c_{i}$ iff $e t_{i u} \geq e t_{i s} \geq s t_{i u}$. The interleaving relation is symmetric but not transitive. A process $p_{s}$ is referred to as connected with another process $p_{u}$ iff (1) $p_{s}$ is interleaved with $p_{u}$ or (2) $p_{s}$ is interleaved with some process $p_{v}$ and $p_{v}$ is connected with $p_{u}$. The connected relation is symmetric and transitive. A schedule sch of a computer $c_{i}$ is a history of processes performed on the computer $c_{i}$. Processes in $s c h_{i}$ are partially ordered in the precedent relation and related in the connected relation. Here, let $K_{i}\left(p_{s}\right)$ be a closure subset of the processes in the schedule $s c h_{i}$ which are connected with a process $p_{s}$, i.e. $K_{i}\left(p_{s}\right)=\left\{p_{u} \mid p_{u}\right.$ is connected with $\left.p_{s}\right\} . K_{i}\left(p_{s}\right)$ is an equivalent class with the connected relation, i.e. $K_{i}\left(p_{s}\right)=K_{i}\left(p_{u}\right)$ for every process $p_{u}$ in $K_{i}\left(p_{s}\right) . K_{i}\left(p_{s}\right)$ is referred to as knot in $s c h_{i}$. The schedule $s c h_{i}$ is divided into knots $K_{i 1}, \ldots, K_{i l_{i}}$ which are pairwise disjointing. Let $p_{u}$ and $p_{v}$ are a pair of processes in a knot $K_{i}\left(p_{s}\right)$ where the starting time $s t_{i u}$ is the minimum and the termination time $e t_{i v}$ is the maximum. That is, the process $p_{u}$ is first performed and the process $p_{v}$ is lastly finished in the knot $K_{i}\left(p_{s}\right)$. The execution time $T K_{i}$ of the knot $K_{i}\left(p_{s}\right)$ is $e t_{i v}-s t_{i u}$. Let $K P_{i}(t)$ be a current knot which is a set of current or previous processes which are connected with at least one current process in $P_{i}(t)$ at time $t$. In the simple model, it is straightforward for the following theorem to hold from the simple model properties:

[Theorem] Let $K_{i}$ be a knot in a schedule $s c h_{i}$ of a computer $c_{i}$. The execution time $T K_{i}$ of the knot $K_{i}$ is $\sum_{p_{s} \in K_{i}} \min T_{i s}$.

Let us consider a knot $K_{i}$ of three processes $p_{1}, p_{2}$, and $p_{3}$ on a computer $c_{i}$ as shown in Figure $3(1)$. Here, $K_{i}=\left\{p_{1}, p_{2}, p_{3}\right\}$. First, suppose that the processes $p_{1}, p_{2}$, and $p_{3}$ are serially performed, i.e. $e t_{i 1}=s t_{i 2}$ and $e t_{i 2}=s t_{i 3}$. Here, the execution time $T K_{i}$ is $e t_{i 3}-s t_{i 1}=m i n T_{i 1}+m i n T_{i 2}+m i n T_{i 3}$. Next, three processes $p_{1}, p_{2}$, and $p_{3}$ start at time $s t$ and terminate at time $e t$ as shown in Figure 3(2). Here, the execution time $T K_{i}$ $=\min _{i 1}+\operatorname{minT}_{i 2}+\operatorname{minT}_{i 3}$. Lastly, let us consider a knot $K_{i}$ where the processes are concurrently performed. The processes $p_{1}, p_{2}$, and $p_{3}$ start at the same time, st $t_{i 1}=$

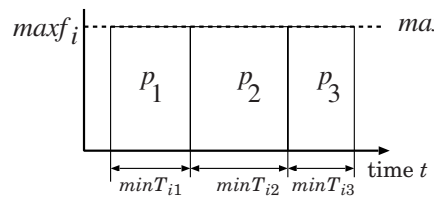

(1) Serial execution.

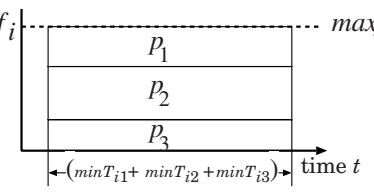

(2) Parallel execution.

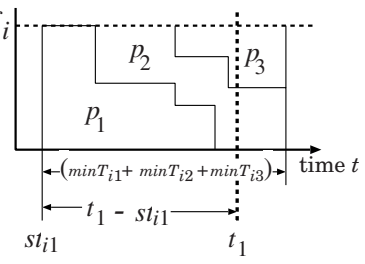

(3) Mixed execution.

Fig. 3. Execution time of knot 
$s t_{i 2}=s t_{i 3}$, are concurrently performed, and the process $p_{3}$ lastly terminates at time $e t_{i 3}$ after $p_{1}$ and $p_{2}$ as shown in Figure 3 (3). Here, the execution time $T K_{i}$ of the knot $K_{i}$ is $e t_{i 3}-s t_{i 1}=m i n T_{i 1}+\operatorname{minT}_{i 2}+\operatorname{minT}_{i 3}$. The current knot $K P_{i}\left(t_{1}\right)$ is $\left\{p_{1}, p_{2}, p_{3}\right\}$ at time $t_{1}$ and $K P_{i}\left(t_{2}\right)$ is $\left\{p_{1}, p_{2}\right\}$ at time $t_{2}$.

It depends on the scheduling algorithm how much each NCR $f_{i s}(t)$ is in the equation (6), $f_{i s}(t)=\alpha_{i s} \cdot \max f_{i}$ where $\sum_{p_{s} \in P_{i}(t)} \alpha_{i s}=1$. In the fair scheduler, each $f_{i s}(t)$ is the same as the others, i.e. $\alpha_{i s}=1 /\left|P_{i}(t)\right|$ :

$$
f_{i s}(t)=\max f_{i} /\left|P_{i}(t)\right| \text {. }
$$

\subsection{Estimated Termination Time}

Suppose there are a set $P$ of processes $\left\{p_{1}, \ldots, p_{m}\right\}$ and a set $C$ of computers $\left\{c_{1}, \ldots\right.$, $\left.c_{n}\right\}$ in a system $S$. We discuss how to allocate a process $p_{s}$ in the process set $P$ to a computer $c_{i}$ in the computer set $C$. Here, we assume the system $S$ to be heterogeneous, i.e. some pair of computers $c_{i}$ and $c_{j}$ have different specifications and performance.

Suppose a process $p_{s}$ is started on a computer $c_{i}$ at time $s t_{i s}$. A set $P_{i}(t)$ of current processes are being performed on a computer $c_{i}$ at time $t$.

[Computation model] Let $K P_{i}(t)$ be a current knot $=\left\{p_{i 1}, \ldots, p_{i l_{i}}\right\}$ of processes, where the starting time is $s t$. The total execution time $T(s t, t)$ of processes in the current knot $K P_{i}(t)$ is given as;

$$
T(s t, t)=\operatorname{minT}_{i 1}+\operatorname{minT}_{i 2}+\cdots+\operatorname{minT}_{i l_{i}}
$$

In Figure 3 (3), $t_{1}$ shows the current time. A process $p_{1}$ is first initiated at time $s t_{i 1}$ and is terminated before time $t_{1}$ on a computer $c_{i}$. A pair of processes $p_{2}$ and $p_{3}$ are currently performed at time $t_{1}$. Here, $K P_{i}(t)$ is a current knot $\left\{p_{1}, p_{2}, p_{3}\right\}$ at time $t_{1}$. $T\left(s t_{i 1}, t_{1}\right)=\min T_{i 1}+\min T_{i 2}+\operatorname{minT}_{i 3}$. The execution time from time $s t_{i 1}$ to $t_{1}$ is $t_{1}$ - $s t_{i 1}$. At time $t_{1}$, we can estimate that the processes $p_{2}$ and $p_{3}$ which are concurrently performed and terminate at the time $t_{1}+T\left(s t_{t 1}, t_{1}\right)-\left(t_{1}-s t_{i 1}\right)=s t_{i 1}+T\left(t_{i 1}, t_{1}\right) . s t_{i 1}$ is referred to as starting time of the current knot $K P_{i}(t)$. No process is performed in some period before $s t_{i 1}$ and some process is performed at any time since $s t_{i 1}$ to $t$.

The estimated termination time $E T_{i}(t)$ of current processes on a computer $c_{i}$ means time when every current process of time $t$ terminates if no other process would be performed after time $t . E T_{i}(t)$ is given as follows:

$$
E T_{i}(t)=t+T\left(s t_{i s}, t\right)-\left(t-s t_{i s}\right)=s t_{i s}+T\left(s t_{i s}, t\right)
$$

Suppose a new process $p_{s}$ is started at current time $t$. By using the equation (9), we can obtain the estimated termination time $E T_{i}(t)$ of the current processes on each computer $c_{i}$ at time $t$. From the computation model, the estimated termination time $E T_{i s}(t)$ of a new process $p_{s}$ starting on a computer $c_{i}$ at time $t$ is given as follows:

$$
E T_{i s}(t)=E T_{i}(t)+\min T_{i s}
$$




\section{Simple Power Consumption Model}

Suppose there are $n(\geq 1)$ computes $c_{1}, \ldots, c_{n}$ and $m(\geq 1)$ processes $p_{1}, \ldots, p_{m}$. In this paper, we assume the simple computation model is taken for each computer, i.e. the maximum clock frequency to be stable for each computer $c_{i}$. Let $E_{i}(t)$ show the electric power consumption of a computer $c_{i}$ at time $t[\mathrm{~W} / \mathrm{tu}](i=1, \ldots, n) . \max E_{i}$ and $\min E_{i}$ indicate the maximum and minimum electric power consumption of a computer $c_{i}$, respectively. That is, $\min E_{i} \leq E_{i}(t) \leq \max E_{i} \cdot \max E$ and $\min E$ show $\max \left(\max E_{1}\right.$, $\left.\ldots, \max E_{n}\right)$ and $\min \left(\min E_{1}, \ldots, \min E_{n}\right)$, respectively. Here, $\min E_{i}$ shows the power consumption of a computer $c_{i}$ which is in idle state.

We define the normalized power consumption rate $(N P C R) e_{i}(t)[1 / \mathrm{tu}]$ of a computer $c_{i}$ at time $t$ as follows:

$$
e_{i}(t)=E_{i}(t) / \max E(\leq 1) .
$$

Let mine $_{i}$ and $\operatorname{maxe}_{i}$ show the maximum power consumption rate $\min _{i} / \max E$ and the minimum one $\max E_{i} / \max E$ of the computer $c_{i}$, respectively. If the fastest computer $c_{i}$ maximumly spends the electric power with the maximum clock frequency, $e_{i}(t)=\max _{i}=1$. In the lower-speed computer $c_{j}$, i.e. $\max f_{j}<\max f_{i}, e_{j}(t)=\max e_{j}$ $<1$.

We propose two types of power consumption models for a computer $c_{i}$, simple and multi-level models. In the simple model, the NPCR $e_{i}(t)$ is given depending on how many number of processes are performed as follows:

$$
e_{i}(t)=\left\{\begin{array}{l}
\text { maxe }_{i} \text { if } N_{i}(t) \geq 1 . \\
\text { mine }_{i} \text { if otherwise }
\end{array}\right.
$$

This means, if one process is performed on a computer $c_{i}$, the electric power is maximally consumed on the computer $c_{i}$. Even if more than one process is performed, the maximum power is consumed in a computer $c_{i}$. A personal computer with one CPU satisfies the simple model as discussed in the experiments of the succeeding section.

The total normalized power consumption $T P C_{i}\left(t_{1}, t_{2}\right)$ of a computer $c_{i}$ from time $t_{1}$ to time $t_{2}$ is given as follows:

$$
\operatorname{TPC}_{i}\left(t_{1}, t_{2}\right)=\int_{t_{1}}^{t_{2}} e_{i}(t) d t
$$

Next, $T P C_{1} \cdot\left(t_{1}, t_{2}\right) \leq t_{2}-t_{1}$. In the fastest computer $c_{i}, T P C_{1} \cdot\left(t_{1}, t_{2}\right)=\operatorname{maxe}_{i} \cdot\left(t_{2}-\right.$ $\left.t_{1}\right)=t_{2}-t_{1}$ if at least one process is performed at any time from $t_{1}$ to $t_{2}$ in the simple model. Let $K_{i}$ be a knot of a computer $c_{i}$ whose starting time is $s t_{i}$ and termination time is $e t_{i}$. The normalized total power consumption of the computer $c_{i}$ to perform every process in the knot $K_{i}$ is $T P C_{i}\left(s t_{i}, e t_{i}\right)$. In the simple model, $T P C_{i}\left(s t_{i}, e t_{i}\right)=$ $\int_{s t_{i}}^{e t_{i}} \operatorname{maxe}_{i} d t=\left(e_{i}-s t_{i}\right) \cdot \operatorname{maxe}_{i}=\sum_{p_{s} \in K_{i}} \operatorname{minT}_{i s} \cdot \operatorname{maxe}_{i}$.

\section{Process Allocation Algorithms}

\subsection{Round-Robin Algorithms}

We consider two types of algorithms, weighted round robin (WRR) [20] and weighted least connection (WLC) [21] algorithms. For each of the WRR and WLC algorithms, 
we consider two cases, Per (performance) and Pow (power). In Per the weight is given in terms of the performance ratio of the servers. That is, the higher performance a server supports, the more number of processes are allocated to the server. In Pow, the weight is defined in terms of the power consumption ratio of the servers. The less power a server consumes, the more number of processes are allocated to the server.

\subsection{Laxity-Based Algorithm}

Some application has the deadline constraint $T C_{s}$ on a process $p_{s}$ issued by the application, i.e. a process $p_{s}$ has to terminate until the deadline. Here, a process $p_{s}$ has to be allocated to a computer $c_{i}$ so that the process $p_{s}$ can terminate by the deadline $T C_{s} \cdot \mathbf{C}_{s}(t)$ denotes a set of computers which satisfy the condition $T C_{s}$, i.e. $\mathbf{C}_{s}(t)=$ $\left\{c_{i} \mid E T_{i s}(t) \leq T C_{s}\right\}$. That is, in a computer $c_{i}$ in $\mathbf{C}_{s}(t)$, the process $p_{s}$ is expected to terminate by the deadline $T C_{s}$. Here, if the process $p_{s}$ is allocated to one computer $c_{i}$ in $\mathbf{C}_{s}(t)$, the process $p_{s}$ can terminate before $T C_{s}$.

Next, we assume that a normalized power consumption rate (NPCR) $e_{i}(t)$ of each computer $c_{i}$ is given as equation (12) according to the simple model. We can estimate the total power consumption laxity $l e_{i s}(t)$ of a process $p_{s}$ between time $t$ and $E T_{i s}(t)$ at time $t$ when the process $p_{s}$ is allocated to the computer $c_{i}$ [Figure 4 . $l e_{i s}(t)$ of the computer $c_{i}$ is given as equation (14):

$$
l e_{i s}(t)=\max _{i} *\left(E T_{i s}(t)-t\right)
$$

Suppose a process $p_{s}$ is issued at time $t$. A computer $c_{i}$ in the computer set $C$ is selected for the a process $p_{s}$ with the constraint $T C_{s}$ at time $t$ as follows:

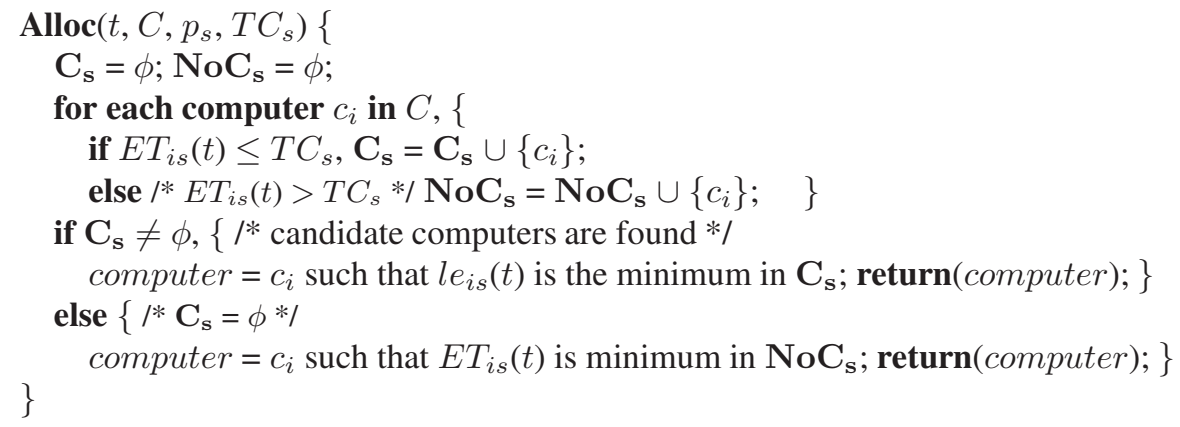

$\mathbf{C}_{\mathbf{s}}$ and $\mathbf{N o C}_{\mathbf{s}}$ are sets of computers which can and cannot satisfy the constraint $T C_{s}$, respectively. Here, $\mathbf{C}_{\mathbf{s}} \cup \mathbf{N o C} \mathbf{C}_{\mathbf{s}}=C$ and $\mathbf{C}_{\mathbf{s}} \cap \mathbf{N o} \mathbf{C}_{\mathbf{s}}=\phi$. In the procedure Alloc, if there is at least one computer which can satisfy the time constraint $T C_{s}$ of process $p_{s}$, one of the computers which consumes the minimum power consumption is selected. If there is no computer which can satisfy the application time constraint $T C_{s}$, one of the computers which can most early terminate the process $p_{s}$ is selected in the computer set $C$. 


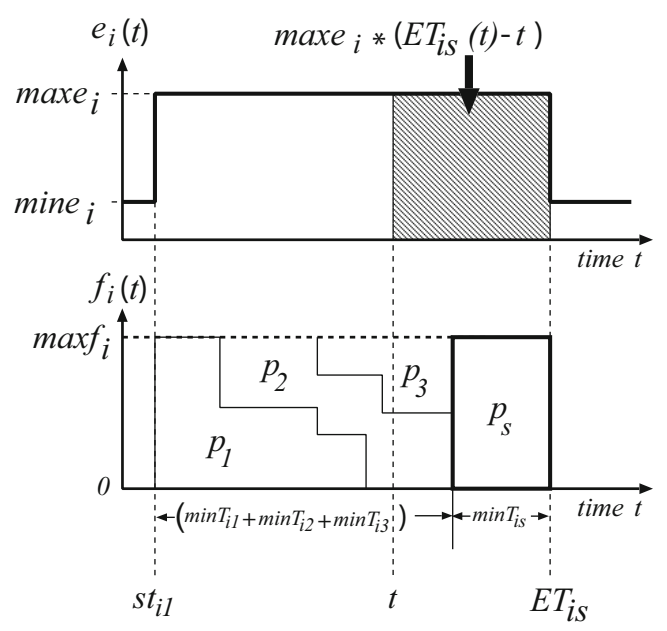

Fig. 4. Estimation of power consumption

\section{Evaluation}

\subsection{Environment}

We measure how much electric power computers consume for Web applications. We consider a cluster system composed of Linux Virtual Server (LVS) systems which are interconnected in gigabit networks as shown in Figure 5 The NAT based routing system VS-NAT [12] is used as the load balancer $K$. The cluster system includes three servers $s_{1}, s_{2}$, and $s_{3}$ in each of which Apache 2.0 [11] is installed, as shown in Figure 5 The load generator server $L$ first issues requests to the load balancer $K$. Then, the load balancer $K$ assigns each request to one of the servers according to some allocation algorithm. Each server $s_{i}$ compresses the reply file by using the Deflate module [13] on receipt of a request from the load generator server $L$.

We measure the peak consumption of electric power and the average response time of each server $s_{i}(i=1,2,3)$. The power consumption ratio of the servers $s_{1}, s_{2}$, and $s_{3}$ is $0.9: 0.6: 1$ as shown in Figure 5 On receipt of a Web request, each server $s_{i}$ finds a

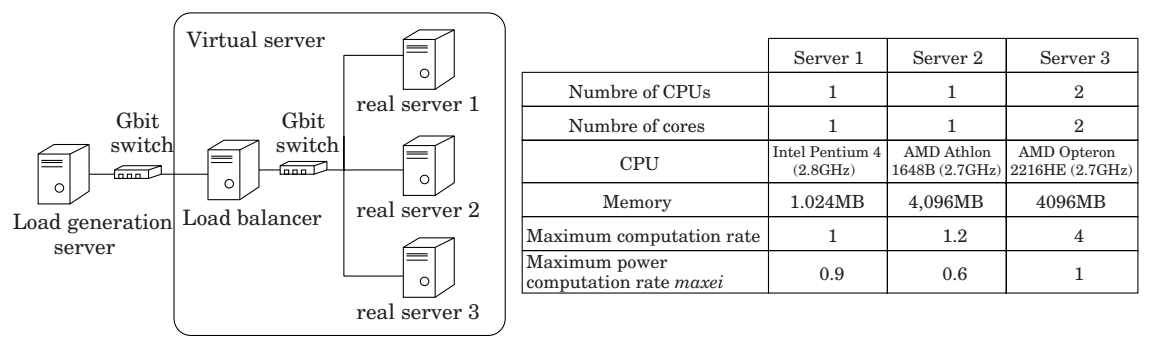

Fig. 5. Cluster system 
reply file of the request and compresses the reply file by using the Deflate module. The size of the original reply file is 1 Mbyte and the compressed reply file is $7.8 \mathrm{Kbyte}$ in size. The Apache benchmark software [10] is used to generate Web requests, where the total number 10,000 of requests are issued where 100 requests are concurrently issued to each server. The performance ratio of the servers $s_{1}, s_{2}$, and $s_{3}$ are $1: 1.2: 4$ as shown in Figure 5] The server $s_{3}$ is the fastest and mostly consumes the electric power. The server $s_{1}$ is slower than $s_{3}$ but more consumes the electric power than the serve $s_{2}$.

\subsection{Experimental Results}

If the weight is based on the performance ratio (Per), the requests are allocated to the servers $s_{1}, s_{2}$, and $s_{3}$ with the ratio $1: 1.2: 4$, respectively. On the other hand, if the weight is based on the power consumption ratio (Pow), the requests are allocated to the servers $s_{1}, s_{2}$, and $s_{3}$ with the ratio $0.9: 0.6: 1$, respectively. Here, by using the Apache benchmark software, the load generation server $L$ transmits totally 100,000 requests to the servers $s_{1}, s_{2}$, and $s_{3}$ where six requests are concurrently issued to the load balancer $K$. The total power consumption of the cluster system and the average response time of a request from a web server are measured. We consider a static web server where the size of a reply file for a request is not dynamically changed, i.e. the compressed version of the same HTML reply file is sent back to each user. In this experiment, the original HTML file and the compressed file are 1,025,027 [Byte] and 13,698 [Byte] in size, respectively. On the load balancer $K$, types of process allocation algorithms are adopted; the weighted round-robin (WRR) [20] algorithms, WRR-Per and WRR-Pow; the weighted least connection (WLC) [21] algorithms, WLC-Per and WLC-Pow.

Figure 6 shows the total power consumption $[\mathrm{W} / \mathrm{H}]$ of the cluster system for time. WRR-Per and WLC-Per show the total power consumption of the servers in the WRR and WLC algorithms with the performance based weight (Per), respectively. WRRPow and WLC-Pow indicate the power consumption of the WRR and WLC with power consumption based weight (Pow), respectively. In WRR-Per and WLC-Per, the total

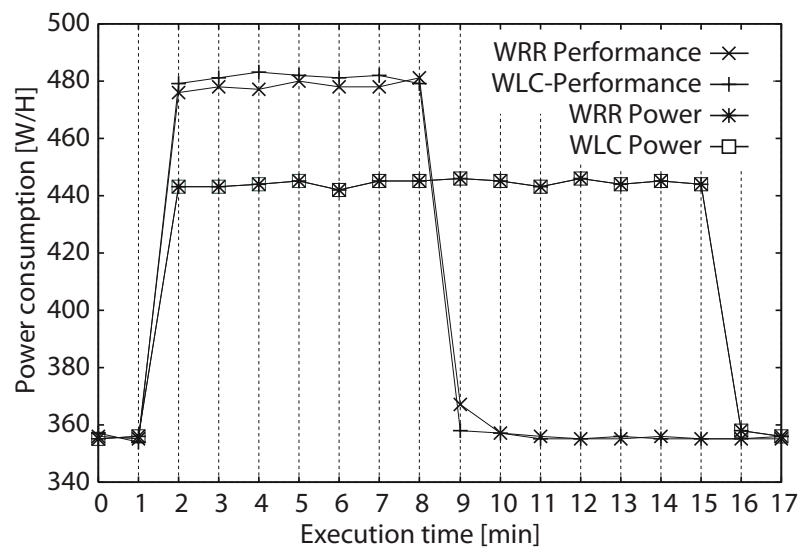

Fig. 6. Power consumption 
execution time and peak power consumption are almost the same. In addition, the total execution time and peak power consumption are almost the same in WRR-Pow and WLC-Pow. This experimental result shows that the total power consumption and total execution time are almost the same for the two allocation algorithms if the same weight ratio is used. If the weight of the load balance algorithm is given in terms of the performance ratio $(\mathrm{Per})$, the peak power consumption is higher than the power consumption ratio (Pow). However, in the Per, the total execution time is longer than Pow. Here, the total power consumption is calculated by the multiplication of the execution time and power consumption. The experiment shows the total power consumption is reduced by using the performance based weight $(\mathrm{Per})$.

\section{Concluding Remarks}

In this paper, we discussed the simple power consumption model of computers. We discussed the laxity-based algorithm to allocate a process to a computer so that the deadline constraint is satisfied and the total power consumption is reduced on the basis of the laxity concept. We obtained experimental results on electric power consumption of Web servers. We evaluated the simple model through the experiment of the PC cluster. Then, we showed the PC cluster follows the simple model. We are now considering types of applications like database transactions and measuring the power consumption of multi-CPU servers.

\section{References}

1. Akyildiz, I.F., Kasimoglu, I.H.: Wireless Sensor and Actor Networks: Research Challenges. Ad Hoc Networks Journal (Elsevier) 2, 351-367 (2004)

2. AMD, http://www. amd. com/

3. Intel, http://www.intel.com/

4. Green IT, http: //www.greenit.net

5. Heath, T., Diniz, B., Carrera, E.V., Meira, W., Bianchini, R.: Energy Conservation in Heterogeneous Server Clusters. In: PPoPP 2005: Proceedings of the tenth ACM SIGPLAN symposium on Principles and Practice of Parallel Programming, pp. 186-195 (2005)

6. Lynch, N.A.: Distributed Algorithms, 1st edn. Morgan Kaufmann Publisher, San Francisco (1997)

7. Montresor, A.: A robust Protocol for Building Superpeer overlay Topologies. In: Proc. of the 4th International Conference on Peer-to-Peer Computing, pp. 202-209 (2004)

8. Bianchini, R., Rajamony, R.: Power and Energy Management for Server Systems. IEEE Computer 37(11) (November 2004); Special issue on Internet data centers

9. Rajamani, K., Lefurgy, C.: On Evaluating Request-Distribution Schemes for Saving Energy in Server Clusters. In: Proc. of the 2003 IEEE International Symposium on Performance Analysis of Systems and Software, pp. 111-122 (2003)

10. ab - Apache HTTP server benchmarking tool, http://httpd.apache.org/docs/2.0/programs/ab.html

11. Apache 2.0, http://httpd.apache.org/

12. VS-NAT, http://www. linuxvirtualserver.org/

13. Apache Module mod-deflate, http://httpd.apache.org 
14. Aron, M., Druschel, P., Zwaenepoel, W.: Cluster Reserves: A Mechanism for Resource Management in Cluster-Based Network Servers. In: Proceedings of the International Conference on Measurement and Modeling of Computer Systems, pp. 90-101 (2000)

15. Bevilacqua, A.: A Dynamic Load Balancing Method on a Heterogeneous Cluster of Workstations. Informatica 23(1), 49-56 (1999)

16. Bianchini, R., Carrera, E.V.: Analytical and Experimental Evaluation of Cluster-Based WWW Servers. World Wide Web Journal 3(4) (December 2000)

17. Heath, T., Diniz, B., Carrera, E.V., Meira Jr., W., Bianchini, R.: Self-Configuring Heterogeneous Server Clusters. In: Proceedings of the Workshop on Compilers and Operating Systems for Low Power (2003)

18. Rajamani, K., Lefurgy, C.: On Evaluating Request-Distribution Schemes for Saving Energy in Server Clusters. In: Proceedings of the IEEE International Symposium on Performance Analysis of Systems and Software, pp. 111-122 (2003)

19. Colajanni, M., Cardellini, V., Yu, P.S.: Dynamic Load Balancing in Geographically Distributed Heterogeneous Web Servers. In: Proceeding of the 18th International Conference on Distributed Computing Systems, p. 295 (1998)

20. Weighted Round Robin (WRR), http: / /www. linuxvirtualserver.org/docs/scheduling.html

21. Weighted Least Connection (WLC), http://www. linuxvirtualserver.org/docs/scheduling.html 